\title{
Endoscopic Surgical Procedures for Fungal Maxillary Sinusitis: How to Do It, a Review
}

\author{
Motohiro Sawatsubashi \\ Department of Otolaryngology-Head and Neck Surgery, Graduate School of Medical Sciences, Kyushu University, \\ Fukuoka, Japan \\ Email: motohiro@qent.med.kyushu-u.ac.jp
}

How to cite this paper: Sawatsubashi, M. (2018) Endoscopic Surgical Procedures for Fungal Maxillary Sinusitis: How to Do It, a Review. International Journal of Otolaryngology and Head \& Neck Surgery, 7, 287-297.

https://doi.org/10.4236/ijohns.2018.75029

Received: July 25, 2018

Accepted: August 31, 2018

Published: September 3, 2018

Copyright (c) 2018 by author and Scientific Research Publishing Inc. This work is licensed under the Creative Commons Attribution International License (CC BY 4.0).

http://creativecommons.org/licenses/by/4.0/

\begin{abstract}
Fungal rhinosinusitis (FRS) is categorized as being either invasive or non-invasive based on the histopathological evidence of tissue invasion by fungi. Endoscopic sinus surgery (ESS) has become the gold standard treatment for non-invasive FRS including sinus fungal ball. It is considered to be an effective and safe procedure. It is important to keep a sufficient field of view in order to remove the fungal debris completely. ESS should also prevent damage to the nasal cavity structures including the inferior turbinate. This report mainly describes the endoscopic surgical procedures for fungal ball of the maxillary sinus (sinus mycelia) based on our methods and review of the literature, including written articles in Japanese. ESS procedures include the middle meatus approach for the maxillary sinus, the combined approach (both middle and inferior meatal antrostomy) for the maxillary sinus, and endoscopic modified medial maxillectomy (EMMM).
\end{abstract}

\section{Keywords}

Fungal Rhinosinusitis, Sinus Fungal Ball, Sinus Mycelia, Endoscopic Sinus Surgery

\section{Introduction}

Fungal rhinosinusitis (FRS) is categorized as either invasive or non-invasive based on the histopathological evidence of tissue invasion by fungi [1]. Invasive FRS includes acute invasive FRS, granulomatous FRS, and chronic invasive FRS [2]. Acute invasive FRS is the most aggressive sinusitis, which shows bone distractions as well as orbit and/or skull base invasion with poor prognosis [3]. Wide resection with high-dose antifungal therapy should be performed immediately for the treatment of acute invasive FRS. Therefore, invasive FRS must be 
distinguished from non-invasive FRS.

In contrast, the non-invasive type grows slowly and in some cases, there are no symptoms [1]. Non-invasive FRS can be divided into saprophytic fungal infestation, fungal ball, and fungus-related eosinophilic FRS including allergic FRS [2]. Fungal ball is the most frequent cause of non-invasive FRS, and the maxillary sinus is the most common location [1] [4].

Endoscopic sinus surgery (ESS) has become the gold standard of treatment or chronic rhinosinusitis, including non-invasive fungal sinusitis. It is considered as an effectiveness and safe procedure, and the recurrence rate is about $5 \%(0 \%$ 22.5\%) [4]. This report mainly describes about endonasal endoscopic surgical procedures for fungal maxillary sinusitis (fungal ball, sinus mycelia), based on our methods and a review of the literature, which included Japanese articles (Table 1).

\section{ESS for Fungal Maxillary Sinusitis}

To treat fungal ball of the maxillary sinus, all of the fungus must be completely removed. To verify complete removal of the fungal ball, it is important to achieve sufficient visualization of the sinus. In addition, the use of not only a $0^{\circ}$ endoscope but also angled endoscopes $\left(30^{\circ}, 45^{\circ}, 70^{\circ}\right)$ are required to achieve this. How to ensure the sufficient visualization? It is sometimes difficult to see the anterior inferior or medial inferior wall of the maxillary sinus even when a $70^{\circ}$ endoscope is used, when the maxillary sinus is observed from a middle meatus window. If $70^{\circ}$ endoscope does not allow sufficient visualization afterwards, then a flexible scope deployed into the maxillary sinus may avoid any unnecessary surgery. Although the surgery involves a large opening of the sinus, including removal of part of the inferior turbinate in some cases, the surgery should also prevent damage to the nasal cavity structures. It is not necessary to make an additional mucosal incision at the canine fossa. It is better to perform the septoplasty prior to ESS in order to achieve sufficient visualization of the sinus in cases with nasal septal deviation. The biopsy of the fungal debris and its surrounding mucosa is necessary to confirm non-invasive FRS when the fungal debris is removed. Nasal and sinus saline irrigation is performed at the end of the surgery.

Table 1. Summarized surgical procedures.

\begin{tabular}{cc}
\hline Surgical procedures & Methods \\
\hline Middle meatus approach & $\begin{array}{c}\text { uncinectomy and middle meatal antrostomy } \\
\text { (standard procure of ESS) }\end{array}$ \\
Combined approach & both middle and inferior meatal \\
Endoscopic modified & antrostomy with preserved inferior turbinate \\
medial maxillectomy (EMMM) & medial maxillectomy with preserved \\
\end{tabular}




\subsection{Middle Meatus Approach}

Endoscopic middle meatal approach is one of the most commonly performed functional ESS procedures. The nasal cavity is decongested using a gauze with lidocaine and epinephrine. The lidocaine with epinephrine is injected at the level of the middle turbinate root and uncinate process. The uncinate process is removed in case of middle meatal antrostomy. Complete removal of fungal debris by ESS through an uncinectomy and a sufficiently wide meatal antrostomy is performed. The large or wide antrostomy means that we have a good visualization of the maxillary sinus and surgical instruments can be inserted easily. There are varying opinions on what the size of the antrostomy should be. If the ethmoid bulla is severely blocked, the ethmoidectomy has to be performed. After widening of the antrostomy for the maxillary sinus, the fungal ball is extracted using curved suctions, forceps, and curved microdebrider blades.

The "Gauze technique" is a simple and quick method used to be removed the fungal ball in the maxillary sinus [5]. It is a standard ESS using traditional instruments with the gauze. The surgical procedure is following the steps. After making a wide antrostomy, the fungus ball is removed with the curved endoscopic pincers and suction. The gaze soaked in a standard saline solution is introduced into the maxillary sinus through the antrostomy. The bulk of the gauze pushes out the remaining fungal debris using a curved suction or curved pincers, and then a gauze can be used to push the fugal ball out of the antrostomy. The same procedure is repeated several times, after which the gaze can be easily removed with curved forceps from the antrostomy.

When fugal debris can be removed completely, it is not necessary to have a large opening in the middle meatus for sufficient access to the maxillary sinus cavity. Although the mucosa should be preserved, it must be biopsied to exclude any possibility of microscopic invasion by fungi. Infected granulation tissue should be removed using curved forceps and microdebrider blades, but the periosteum of the sinus must be preserved in such cases. When the fungal ball is located near the ostiomeatal complex, it is a good indication for a middle meat us approach (Figure 1).

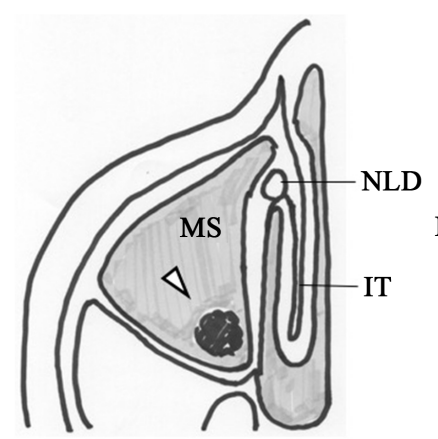

(a)

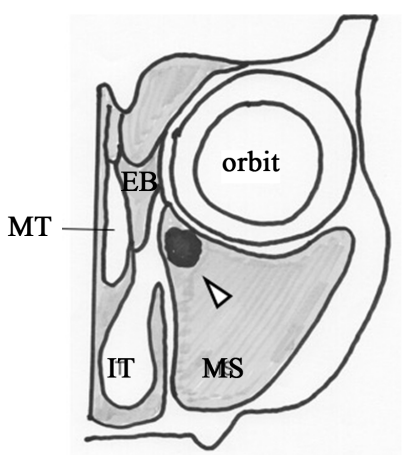

(b)

Figure 1. Schema of Axial (a) and Coronal section (b): Fungus ball (Arrow head) is located near the ostiomeatal complex. NLD, Nasolacrimal duct. IT, Inferior turbinate. MT, Middle turbinate. ES, Ethmoid sinus. MS, Maxillary sinus. 
It is impractical to make a new window at the inferior meatus for these cases. On the other hand, it is sometimes difficult to see the anterior inferior or medial inferior wall of the maxillary sinus even when a $70^{\circ}$ endoscope is used, when the maxillary sinus is observed from a middle meatus window. The insufficient visualization of the entire maxillary sinus means that removal of the fungal ball will be incomplete. Thus, it is possible that fungal debris removal will be incomplete from a middle meatus window, and only when the fungus ball is not located in the anterior inferior and medial inferior wall of the maxillary sinus, will complete removal be achieved.

\subsection{Combined Approach (Both Middle and Inferior Meatal Antrostomy)}

Our department recently published a study that combined both middle and inferior meatal antrostomy. This combination was shown to be effective for the treatment of FRS [6]. Our study showed that the results of ESS with the combined approach were better than the middle meatal approach alone without inferior meatal antrostomy [6]. The post-operative CT examination at 3 to 4 months after the ESS, showed a normal maxillary sinus, and no cases of recurrence were observed in combined approach group in our study (Figure 2) [6]. Klossek et al described that the middle meatus antrostomy had to be associated with an inferior antrostomy in about $65 \%$ of the cases in order to facilitate access
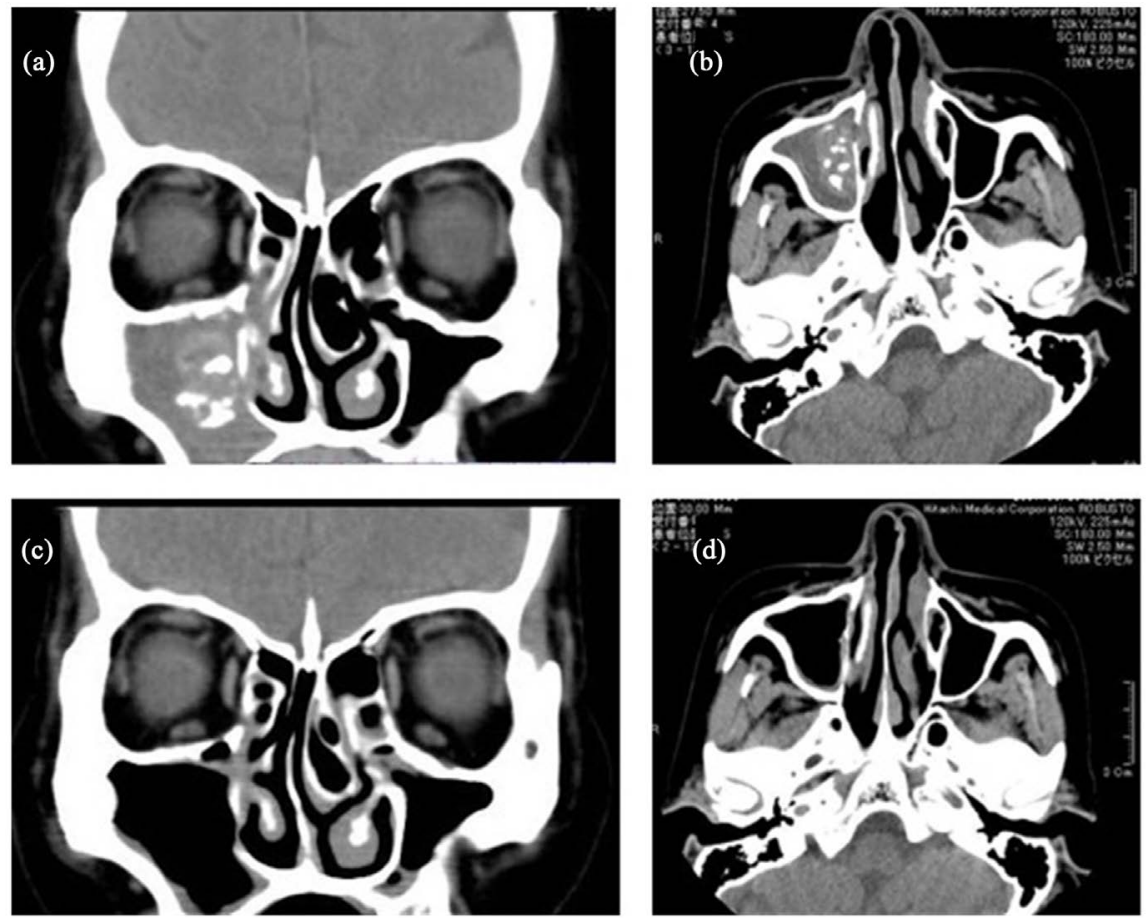

Figure 2. CT examination of a case where the combined method was utilized: Figure 3(a) and Figure 3(b) show soft tissue attenuation with a high-density lesion in the right maxillary sinus. Figure 3(c) and Figure 3(d) show the post-operative CT after ESS with both the middle and the inferior meatal antrostomy. 
and removal of the fungal ball [7]. Dufour et al. reported 150 fungus ball maxillary sinusitis cases that revived the ESS procedure [8]. They performed middle meatus antrostomy $(n=53 / 150,35 \%)$ and a combination of middle and inferior antrostomy ( $\mathrm{n}=97 / 150,65 \%$ ) [8]. Based on these reports, the combination of middle and inferior antrostomy appeared to be required in over $60 \%$ of fungus ball maxillary sinusitis ESS procedures. The double ventilation pathway operation is superior to the single ventilation pathway. The combined approach makes it easier to observe the whole sinus, especially the anterior inferior and medial inferior wall of the maxillary sinus, compared with the single middle meatus approach. As for the post-operative micociliary function of the maxillary sinus, the previous report suggested that a combination of middle and inferior meatal antrostomy does not disturb the microciliary clearance [9]. Our previous study showed that a combination of the middle and inferior meatus antrostomy approaches did not disturb the post-operative nasal condition [6]. We performed the combined approach of ESS in the following steps. First, the uncinate process was removed for a sufficiently wide meatal antrostomy (see 2-1. Middle meatus approach). The surgeon decided whether or not to perform an inferior meatal antrostomy based on the observation of the maxillary sinus which was observed from the middle meatal window. When the fungal ball was located on the anterior or inferior side of the maxillary sinus, or if the surgeon could not see that area, an inferior meatal antrostomy was performed. In this approach, the bridge of bone between the two antrostomy preserved the inferior turbinate. The inferior turbinate is fractured medially, allowing space for the endoscope (0 or 30 degree) prior to make an inferior meatal antrostomy being made.

Even if the inferior meatal antrostomy is closed after the surgery, which is not a major problem, we usually perform the meatal mucosal flap procedure to avoid closure or stenosis of the inferior meatal antrostomy (Figure 3). Tani et al. reported that the inferior meatal antrostomy opening ratio was $89 \%$ for the inferior

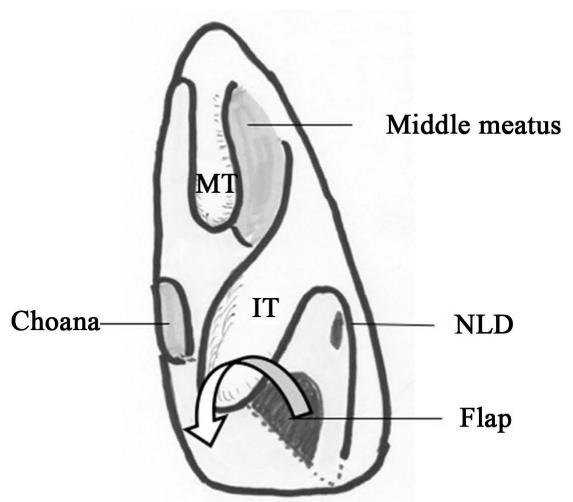

(a)

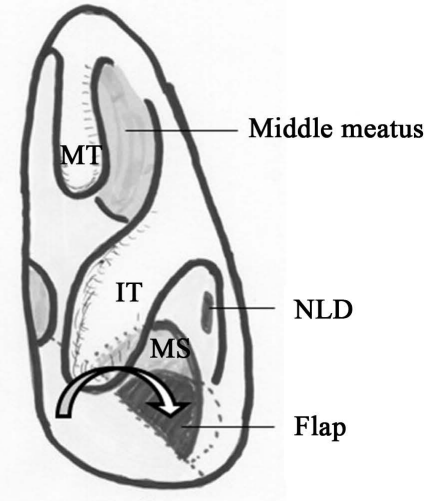

(b)

Figure 3. The inferior meatal mucosal flap procedure. (a) The U-shaped mucosal flap was positioned on the nasal floor after the elevation from the meatal bone; (b) The flap was positioned across the inferior lip of the bony window into the maxillary sinus after removing boney wall. NLD, Nasolacrimal Duct. IT, Inferior Turbinate. MT, Middle Turbinate. 
meatal mucosal flap procedure after ESS [10]. The U shaped meatal flap was elevated on the inferior and lateral mucosa in the inferior meatus. After elevation from the meatal bone, the flap was positioned on the nasal floor (Figure 3(a)). Then an inferior meatal window was created using a perforator, or chisel. The bony wall was removed piece-by-piece to make a sufficient opening in the maxillary sinus. When the maxillary bone is hard, and it is difficult to make a hole, it is better to use an angled drill. After making an opening that was sufficiently wide, the mucosal flap was positioned across the inferior lip of the bony window into the maxillary sinus (Figure $3(b)$ ). We can easily observe the inside of maxillary sinus and insert surgical instruments through the inferior window or middle meatus.

To remove the fungal ball, a gauze which has a size of either $3 \mathrm{~cm} \times 15 \mathrm{~cm}$ or 3 $\mathrm{cm} \times 30 \mathrm{~cm}$ is generally used. The gauze is soaked with normal saline or saline which includes epinephrine. The gauze is introduced into the maxillary sinus from the inferior meatal window using curved forceps (Figure 4(a)). Several gauzes are pushed into the maxillary sinus (Figure 4(b)), and then a gauze can be used to push the fugal ball out of the middle meatus (Figure 4(c)). The gauze can then be removed from the middle meatus or inferior meatal window. The same procedure is repeated until fungal ball can be completely removed (Figure $4(d))$.

Okanoue et al. described the combined approach using a Foley catheter instead of the gauze-assisted technique [11]. They insert the catheter into the maxillary sinus from the inferior meatal antrostomy. The inflated balloon $(10 \mathrm{ml})$ causes fungal debris to extrude into the nasal cavity through the middle meatal antrostomy. Debris may remain on inferior or posterior wall of the maxillary sinus, which can then be removed by curved suction, forceps or curved microdebrider blades. This is easy and effective way to remove a fungal ball.

\subsection{Endoscopic Modified Medial Maxillectomy (EMMM)}

Endoscopic medial maxillectomy with medial sift of preserved inferior turbinate

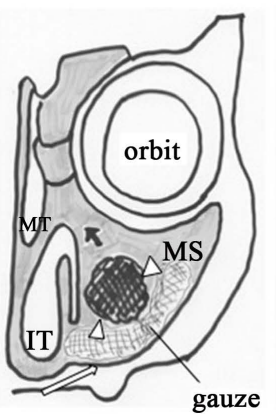

(a)

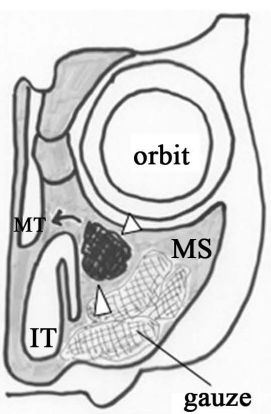

(b)

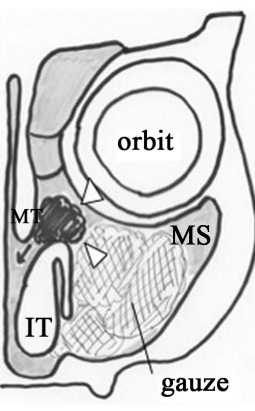

(c)

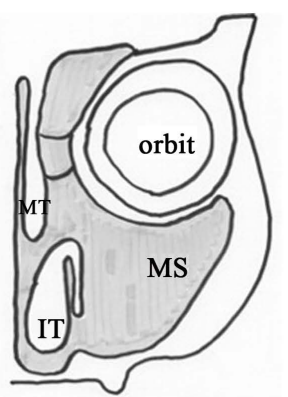

(d)

Figure 4. The combined approach using gauze technique. (a) The approaches (white arrow) are managed through the inferior meatal antrostomy; (b) (c) The gauze pushes Fungal ball (arrow head) out from Maxillary sinus (MS) through the middle meatus; (d) Fungal ball is completely removed. IT, Inferior Turbinate. MT, Middle Turbinate. 
is a procedure for FRS that was introduced in 2008 (Japanese article) [12]. It was described that the head of the inferior turbinate is cut once using turbinate scissors up to the middle meatal antrostomy. This was done so that there is sufficient visualization of the sinus from the inferior meatus. After sifting the preserved inferior turbinate to the posterior midline position, the medial maxillectomy is performed from the middle meatal antrostomy using the backbiter cutting forceps. After the removal of fungal ball, the turbinate is repositioned and sutured with the anterior mucosa in its original position. This method was effective and has been widely accepted in Japan because it utilizes a relatively easy technique with a straight endoscope and instruments from to the inferior meatus. It is similar to the previously reported endoscopic medial maxillectomy with preservation of the inferior turbinate [13] [14]. These approaches are managed through the posterior side of the nasolacrimal duct. Therefore, it is difficult to obtain good visualization of the fungal ball located in the area between the nasolacrimal duct and anterior wall of the maxillary sinus (Figure 5(a)). In such a difficult case, endoscopic modified medial maxillectomy (EMMM) is the preferred choice (Figure 5(b)) [15]. Tsuta et al. first described this effective procedure for chronic sinusitis showing a transnasal endoscopic medial maxillectomy with preserved inferior turbinate and nasolacrimal duct in 1993 (Figure 6)

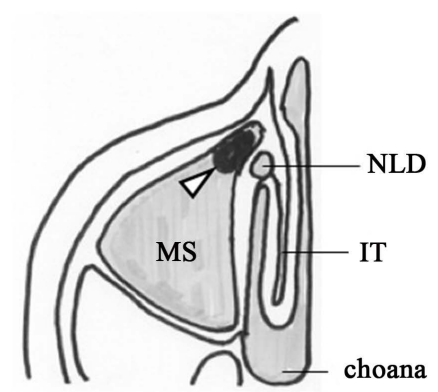

(a)

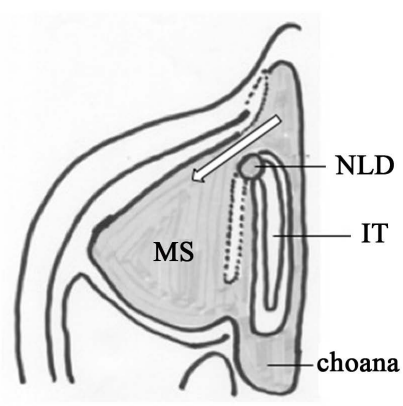

(b)

Figure 5. (a) Fungal ball (arrow head) located in the angle of between the nasolacrimal duct (NLD) and anterior wall of the maxillary sinus; (b) Schema of endoscopic modified medial maxillectomy (EMMM). The approaches are managed through the anterior side of the NLD (white arrow). IT, Inferior turbinate. MS, Maxillary sinus.

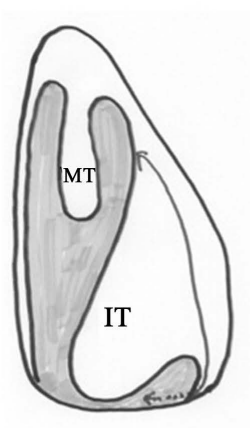

(a)

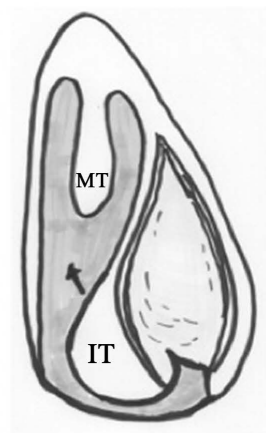

(b)

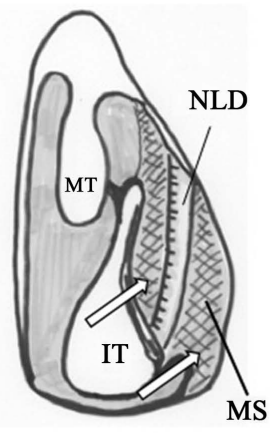

(c)

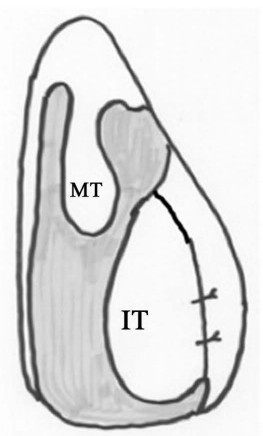

(d)

Figure 6. The endoscopic modified medial maxillectomy (EMMM) procedure. 
[16]. A vertical incision is made in the mucosa of the lateral wall along the anterior margin of the inferior turbinate to the nasal floor (Figure 6(a)). The lateral nasal mucosa is separated from the medial maxillary wall bone (Figure 6(b)). They described that this procedure is initiated by an incision similar to the one used for submucosal resection of the inferior turbinate. After resection of the bone of the inferior turbinate, osteotomy of the medial maxillary bone is performed. At this time, the bone around the nasolacrimal duct is removed gently, and then the nasolacrimal duct is free from the bone (Figure $6(\mathrm{c})$ ). The preserved inferior turbinate mucosa and the nasolacrimal duct can be swung from side to side. The authors called these steps of the procedure "the swing method." After the removal of fungal debris, the turbinate is repositioned and sutured with the anterior mucosa in its original position (Figure 6(d)). This procedure is similar to the modified transnasal endoscopic medial maxillectomy for inverted papilloma published in 2011 [17]. When it is necessary to obtain improved access to the maxillary sinus, osteotomy of the anterior wall of the maxillary sinus can be added. These approaches have become widely accepted and recognized as EMMM (we call it EM-three) for FRS.

\section{Using Powered Devices}

An electrically powered, cylindrical shaver (vacuum-powered microdebrider) can use continuous suction to remove fungal debris and the related granulation as well as polypoid tissue [18]. The prevent blades are available in a variety of angles. It is better to use the cylindrical shaver after fungal ball debulking. Because these often clog with the sticky fungal material.

A powered saline sinus irrigation system (for example, Hydrodebrider System) will help the removal of the fungal ball or bacterial colonies from the paranasal sinuses [19] [20]. The handpiece delivers a rotating spray of pressurized saline with incorporated suction. The pressured saline spray directly cleans the sinus with angles of 0 to 270 degrees [20].

\section{Complications}

As with any endoscopic surgery procedure, ESS has associated risks. Although the risk of a complication of ESS for FRS is low, it is important to understand the potential complications. In most studies, complications of ESS for FRS were the same as those described of Functional ESS for sinusitis [1]. According to the review article, minor complications totaled 5\%, and major complications were around $0.5 \%-1 \%$ for ESS [21]. The most common complication is epistaxis, mainly due to injury of the posterior nasal branch of the sphenopalatine artery [1]. In chronic rhinosinusitis cases, the most common complications were postoperative hemorrhage ( $1.2 \%, 41$ of 3402 cases), cerebrospinal fluid leak $(0.56 \%$, 19 of 3402 cases), and orbital complications ( $0.85 \%, 29$ of 3402 cases) [22]. Before ESS, we should pay attention to certain risk factors and preliminary damage. We must understand the risk factors prior to ESS being performed. There- 
fore, it is very important to perform adequate preoperative imaging. We must check the uncinate process attachment zone, medial orbital wall, skull base, cribriform, plate, anterior ethmoid artery, sphenopalatine artery, optic canal, internal carotid artery and so on before ESS [21].

\section{Post-Operative Care}

Post-operative care is similar to that used after functional ESS for sinusitis. Previous studies have shown that non-invasive FRS does not require adjuvant local or systemic antimycotic chemotherapy after ESS. The patients require nasal and sinus saline irrigation using a bulb syringe after ESS [1]. The short-term use of steroid drops or spray will be effective for treating the edema of the rhinosinus mucosa [1]. The follow-up examination can involve either an endoscopic nasal or CT examination. We performed the post-operative CT examination three or four months after ESS, in order to confirm the patient had healed [6].

\section{Conclusion}

ESS is the standard treatment for non-invasive FRS including sinus fungal ball. All of the fungus must be completely removed. To verify complete removal of the fungal ball, it is important that there is sufficient visualization of the sinus. We have considered useful approaches such as, the middle meatal approach, combined middle and inferior meatal approach, and endoscopic medial maxillectomy, endoscopic modified medial maxillectomy (EMMM) for fungal maxillary sinusitis (Figure 7). These approaches are summarized in Table 1. Furthermore, we must understand the risks of these approaches to avoid the complications of ESS.

\section{Acknowledgements}

I would like to thank Editage (http://www.editage.jp) for their English language editing.

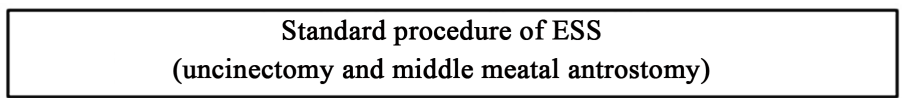

Middel meatus approach

Incomplete removal of the fungus ball and related tissue
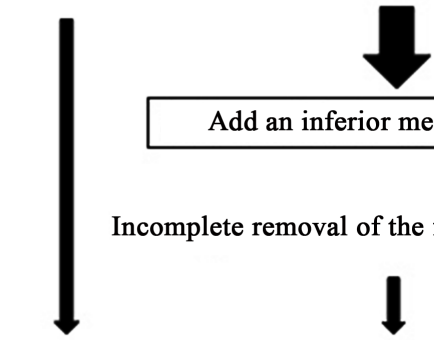

Add an inferior meatal antrostomy

Combined approach

Incomplete removal of the fungus ball and related tissue

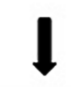

Add modified medial maxillectomy

EMMM

Figure 7. Flow chart of ESS for FRS. This flow shows an example. The following points are important to select ESS procedures. 1) Sufficient visualization of the sinus. 2) Complete removal of the fungal ball with related tissue. 3) Avoid any unnecessary surgery. 


\section{Conflicts of Interest}

The author declares no conflicts of interest regarding the publication of this paper.

\section{References}

[1] Grosjean, P. and Weber, R. (2007) Fungus Balls of the Paranasal Sinuses: A Review. European Archives of Oto-Rhino-Laryngology, 264, 461-470. https://doi.org/10.1007/s00405-007-0281-5

[2] Chakrabarti, A., Denning, D.W., Ferguson, B.J., Ponikau, J., Buzina, W., Kita, H., Marple, B., Panda, N., Vlaminck, S., Kauffmann-Lacroix, C., Das, A., Singh, P., Taj-Aldeen, S.J., Kantarcioglu, A.S., Handa, K.K., Gupta, A., Thungabathra, M., Shivaprakash, M.R., Bal, A., Fothergill, A. and Radotra, B.D. (2009) Fungal Rhinosinusitis: A Categorization and Definitional Schema Addressing Current Controversies. Laryngoscope, 119, 1809-1818. https://doi.org/10.1002/lary.20520

[3] Ergun, O., Tahir, E., Kuscu, O., Ozgen, B. and Yilmaz, T. (2017) Acute Invasive Fungal Rhinosinusitis: Presentation of 19 Cases, Review of the Literature, and a New Classification System. Journal of Oral and Maxillofacial Surgery, 75, 767.e1-767.e9. https://doi.org/10.1016/j.joms.2016.11.004

[4] Pagella, F., Matti, E., De Bernardi, F., Semino, L., Cavanna, C., Marone, P., Farina, C. and Castelnuovo, P. (2007) Paranasal Sinus Fungus Ball: Diagnosis and Management. Mycoses, 50, 451-456. https://doi.org/10.1111/j.1439-0507.2007.01416.x

[5] Garofalo, P., Griffa, A., Dumas, G. and Perottino, F. (2016) “Gauze Technique” in the Treatment of the Fungus Ball of the Maxillary Sinus: A Technique as Simple as It Is Effective. International Journal of Otolaryngology, 2016, Article ID: 4169523. https://doi.org/10.1155/2016/4169523

[6] Katsuobushi, M., Murakami, D., Umezaki, T. and Komune, S. (2015) Endonasal Endoscopic Surgery with Combined Middle and Inferior Meatal Antrostomies for Fungal Maxillary Sinusitis. The Journal of Laryngology \& Otology, 129, S52-S55. https://doi.org/10.1017/S0022215114002394

[7] Klossek, J.M., Serrano, E., Péloquin, L., Percodani, J., Fontanel, J.P. and Pessey, J.J. (1997) Functional Endoscopic Sinus Surgery and 109 Mycetomas of Paranasal Sinuses. Laryngoscope, 107, 12-17. https://doi.org/10.1097/00005537-199701000-00021

[8] Dufour, X., Kauffmann-Lacroix, C., Ferrie, J.C., Goujon, J.M., Rodier, M.H., Karkas, A. and Klossek, J.M. (2005) Paranasal Sinus Fungus Ball and Surgery: A Review of 175 Cases. Rhinology, 43, 34-39.

[9] Ochi, K., Sugiura, N., Komatsuzaki, Y., Nishino, H. and Ohashi, T. (2003) Patency of Inferior Meatal Antrostomy. Auris Nasus Larynx, 30, S57-S60. https://doi.org/10.1016/S0385-8146(02)00146-3

[10] Tani, A., Tada, Y., Miura, T., Suzuki, T., Nomoto, M., Saijo, H., Ono, M., Ogawa, H. and Omori, K. (2011) Surgical Approach for Maxillary Fungal Sinusitis. Japanese Journal of Rhinology, 50, 26-30. (Article in Japanese with English Abstract) https://doi.org/10.7248/jjrhi.50.26

[11] Okanoue, Y., Shoji, K., Hori, R., Hamaguchi, K., Morita, M., Fujimura, S. and Wakizaka, H. (2013) Ingenuity in Endoscopic Surgery for Non-Invasive Fungal Maxillary Sinusitis. Journal of Japan Society for Head and Neck Surgery, 23, 61-64. (Article in Japanese with English Abstract)

[12] Matsune, S. (2008) Endoscopic Sinus Surgery for Maxillary Mycosis. Practica 
Oto-Rhino-Laryngologica (Kyoto), 101, 826-827. (Article in Japanese) https://doi.org/10.5631/jibirin.101.826

[13] Weber, R.K., Werner, J.A. and Hildenbrand, T. (2010) Endonasal Endoscopic Medial Maxillectomy with Preservation of the Inferior Turbinate. American Journal of Rhinology \& Allergy, 24, 132-135. https://doi.org/10.2500/ajra.2010.24.3531

[14] Gras-Cabrerizo, J.R., Massegur-Solench, H., Pujol-Olmo, A., Montserrat-Gili, J.R., Ademá-Alcover, J.M. and Zarraonandia-Andraca, I. (2011) Endoscopic Medial Maxillectomy with Preservation of Inferior Turbinate: How Do We Do It? European Archives of Oto-Rhino-Laryngology, 268, 389-392. https://doi.org/10.1007/s00405-010-1347-3

[15] Nomura, K., Ikushima, H., Ozawa, D., Shimizu, Y., Arakawa, K., Suzuki, J., Hidaka, H., Katori, Y. and Ohyama, K. (2018) Endoscopic Modified Medial Maxillectomy for Fungal Ball of the Hypoplastic Maxillary Sinus with Bony Hypertrophy. Journal of Craniofacial Surgery, 29, e304-e307. https://doi.org/10.1097/SCS.0000000000004379

[16] Tsuta, Y., Murata, K., Minamitani, H. and Ohta, F. (1993) Transsubmucosal Maxilloethmoid Sinus Surgery through Inferior Turbinate (Swinging of Nasolacrimal Duct and Inferior Turbinate). Nihon Jibiinkoka Gakkai Kaiho, 96, 1902-1906. https://doi.org/10.3950/jibiinkoka.96.1902

[17] Suzuki, M., Nakamura, Y., Nakayama, M., Inagaki, A., Murakami, S., Takemura, K. and Yokota, M. (2011) Modified Transnasal Endoscopic Medial Maxillectomy with Medial Shift of Preserved Inferior Turbinate and Nasolacrimal Duct. Laryngoscope, 121, 2399-4201. https://doi.org/10.1002/lary.22326

[18] Mirante, J.P., Krouse, J.H., Munier, M.A. and Christmas, D.A. (1998) The Role of Powered Instrumentation in the Surgical Treatment of Allergic Fungal Sinusitis. Ear, Nose \& Throat Journal, 77, 678-680, 682.

[19] Doi, K. and Nibu, K. (2013) Endoscopic Sinus Surgery with Hydrodebrider System-In Use Experience for Maxillayu Sinus Mycetoma. Oto-Rhino-Laryngology Tokyo, 56, 300-302.

[20] Lo, W.C., Liao, L.J., Wang, C.T. and Cheng, P.W. (2013) Powered Saline Irrigation Is Useful for Endoscopic Removal of Fungus Balls in Maxillary Sinuses: Our Experience in Fifty Patients. Clinical Otolaryngology, 38, 165-169. https://doi.org/10.1111/coa.12066

[21] Hosemann, W. and Draf, C. (2013) Danger Points, Complications and Medico-Legal Aspects in Endoscopic Sinus Surgery. GMS Current Topics in Otorhinolaryngology_Head and Neck Surgery, 12, Doc06.

[22] Stankiewicz, J.A., Lal, D., Connor, M. and Welch, K. (2011) Complications in Endoscopic Sinus Surgery for Chronic Rhinosinusitis: 25-Year Experience. Laryngoscope, 121, 2684-2701. https://doi.org/10.1002/lary.21446 